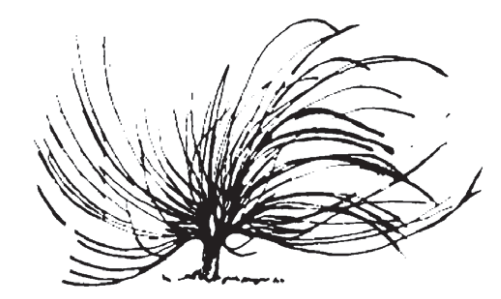

\title{
Caracterización de la Problemática en los Procesos de Enseñanza y Aprendizaje de la Física en Secundaria
}

\author{
Carolina Carpio Chavarría \\ Universidad Estatal a Distancia \\ San José, Costa Rica \\ caricarpio@gmail.com
}

\begin{abstract}
Resumen
El desarrollo del artículo pretende establecer que algunas de las causas de la problemática del aprendizaje de la Física en secundaria tienen una estrecha relación con la metodología tradicionalista predominante en los procesos de enseñanza y aprendizaje de esta materia. El artículo se deriva del proyecto de graduación para nivel de maestría Diseño y producción del curso en línea "Aprendiendo a enseñar Física: hacia un cambio en la labor docente" apoyado con un material didáctico multimedial. Se realizó un análisis teórico, así como una pequeña investigación tipo sondeo con estudiantes, docentes y especialistas en el tema para caracterizar la problemática y fundamentar la relación que existe entre las dificultades que un estudiante pueda tener en su proceso de aprendizaje de la Física y la manera que el docente desarrolla las lecciones. Además se evidencia que la formación universitaria de los y las docentes en Ciencias Naturales requiere actualizarse y prestar mayor atención a aspectos de didáctica
\end{abstract}

1 Recibido: 23 de setiembre de 2013 - Aprobado: 22 de enero de 2014

Tecnóloga educativa dedicada al desarrollo de proyectos e-learning y capacitación docente a nivel universitario. Docente en la enseñanza de las Ciencias Naturales, con experiencia en docencia universitaria y secundaria. Magíster en Tecnología Educativa con énfasis en la producción de medios instruccionales de la Universidad Estatal a Distancia, Costa Rica. 
y mediación pedagógica específicas para esta ciencia, así como a la utilización de tecnologías para apoyar el aprendizaje en Física. Las necesidades de los educandos deben ser atendidas promoviendo mayor dinamismo y participación; aprovechando las múltiples aplicaciones web, que facilitan los procesos de exploración y experimentación de los contenidos. Todo esto con el objetivo de promover un aprendizaje significativo por parte de los y las estudiantes, así como aumentar su motivación e interés por esta ciencia.

Palabras claves: enseñanza de la física, aprendizaje de la física, ciencias naturales, educación secundaria, educación tradicionalista

\begin{abstract}
The object of study of this article is to show how some of the causes associated with the disappointing results of the learning of physics in high school have a close relationship with the dominant traditionalist methodology in the teaching of this subject. This article comes from a master's graduation project titled "Diseño y producción del curso en línea 'Aprendiendo a enseñar Física: Hacia un cambio en la labor docente' apoyado con un material didáctico multimedial." A theoretical analysis was done, and a survey instrument was conducted with a small sample of students, teachers, and some specialists to describe the current situation. This activity corroborated the relationship between difficulties a student may have when learning physics and the way teachers teach. There are deficiencies that make high-school natural science teachers not to be adequately prepared to teach the subject. It is necessary for universities to review and update their programs, paying careful attention to specific didactic and pedagogic mediation as well as the use of technology to support physics instruction. Therefore, teachers can come up with effective practices and innovative methods to teach high-school students. Natural science instruction should establish the construction of concepts by concrete experiences, experiments, and activities for exploration. Therefore, classes should become learning dynamic environments where students have the chance
\end{abstract}


to participate actively, taking advantage of technological tools to achieve curricular objectives. All this can positively affect the quality of instruction in high-schools, promote a significant learning, and improve motivation and interest in natural science.

Keywords: physics teaching, physics learning, education, traditionalist education, natural science

\section{Introducción}

L

a Física es una ciencia significativa e influyente que nace de la observación de la naturaleza; por ello, es natural pensar que para quienes han estudiado las leyes y los principios de la Física, resulta fácil encontrar las relaciones entre éstos y el mundo que los rodea. Sin embargo, es conocido que no siempre sucede así para los estudiantes de secundaria, quienes en muchas ocasiones no logran enlazar lo que se les enseña en las clases de Física, con los fenómenos naturales y las diversas situaciones que diariamente observan.

También, se podría pensar que los estudiantes advierten que los conocimientos adquiridos en la materia les serán de mucha utilidad para su vida cotidiana y en un futuro como profesionales, pues la Física tiene una estrecha relación con las demás ciencias. Sin embargo, el panorama general parece indicar que una importante cantidad de estudiantes de secundaria presentan dificultades en el proceso de aprendizaje de esta ciencia y no encuentran su verdadera utilidad.

Resulta lógico afirmar que son múltiples las razones y situaciones que pueden afectar en esta problemática, dado que los procesos de aprendizaje en general, dependen de muchos factores; para cada persona existen situaciones particulares que contribuyen o dificultan sus procesos educativos formales. Sin embargo, algunos de los factores que influyen de forma directa en esta problemática que enfrentan los procesos de enseñanza y aprendizaje en Física, están en estrecha relación con el accionar de los docentes. Por ejemplo, la metodología y las estrategias didácticas o de mediación que se utilicen para desarrollar las clases.

Caracterizar algunas de las razones específicamente relacionadas con la labor docente es el propósito del presente artículo, el cual se deriva del proyecto titulado Diseño y producción del curso en línea 
"Aprendiendo a enseñar Física: hacia un cambio en la labor docente" apoyado con un material didáctico multimedial. Este proyecto fue presentado como trabajo final de graduación para optar por el grado de Magíster en Tecnología Educativa con énfasis en la producción de medios instruccionales en la Universidad Estatal a Distancia.

Para la justificación y fundamentación del proyecto se realizó un análisis teórico, basado en bibliografía reciente de diversos autores reconocidos en el tema, para contextualizar el panorama de la situación de la enseñanza de la Física e identificar el problema de interés. Este análisis evidencia que la problemática de los procesos enseñanza y aprendizaje de esta ciencia ha sido detectada, definida y analizada por diversos físicos y educadores en todo el mundo.

Además, fue necesario realizar una pequeña investigación tipo sondeo, con el objetivo de fundamentar el problema específicamente en Costa Rica, pues la bibliografía sobre esta problemática en el país es escasa, muy enfocada a artículos de opinión y noticias.

\section{Estado de la cuestión: Panorama de la enseñanza de la Física}

La Física es una de las áreas de saber en la que, en muchos contextos, aún se enseña con metodologías tradicionalistas. Esto es un hecho que ha preocupado a la educación en esta ciencia mundialmente.

Por ejemplo, Carl E. Wieman, luego de ganar el Nóbel de Física en 2002, se ha dedicado a atender problemas de enseñanza y aprendizaje en el área. Al respecto, Wieman (citado en Picquart, 2007) advierte que:

La enseñanza debe de ser más científica en el sentido que las teorías sobre los procesos de aprendizaje, y lo mejor que pueden aportar debe de basarse en mediciones cuantitativas. [...] Muchos profesores adoptan la actitud opuesta, justificando su estilo de enseñanza en base a uno o dos comentarios positivos que reciben de estudiantes. Estos colegas deberían de pensarlo bien ya que todos los resultados de la investigación en ciencia de la educación van en la misma dirección: los estudiantes aprenden muy poco en cursos tradicionales. (p. 30). 
Lo más preocupante aquí para la educación en Ciencias, y en particular de la Física, según Picquart (2007), es que

(...) los trabajos de investigación en didáctica de los últimos veinticinco años muestran que la enseñanza tradicional, por lo esencial la clase magistral, que considera que los alumnos son vasos vacíos que el profesor tiene que llenar, ha fallado en todos los países del mundo en particular en los primeros años de la universidad. (p.30).

Desde el panorama latinoamericano, Mora (2007) expone sobre la educación tradicionalista y las desventajas que produce:

La educación tradicionalmente se ha apoyado en un modelo de enseñanza de exposición de clase-conferencia por el profesor y la memorización de textos por el alumno con el fin de presentar exámenes. En este proceso, el conocimiento es un guión elaborado, administrado por el maestro, y transmitido a los alumnos por diferentes medios por lo general en forma pasiva. Las clases son escenarios serios, silenciosos y con actividades individuales con interacción directa con el docente e indirecta con los mismos alumnos. Entre las limitaciones de este modelo se tiene que una vez terminados los estudios, los alumnos tienen pocas posibilidades de diversificar el conocimiento, pues a lo largo de su formación se han sometido a lo que marca el programa de estudios. Entre las desventajas de este modelo se tiene: i) La adquisición de datos e información a corto plazo. ii) Habilidades limitadas a sus necesidades inmediatas no planificadas en el currículo. iii) Valores individualistas y concepción limitada del trabajo en equipo. iv) La dependencia total hacia una figura de autoridad (maestro). v) Una actitud pasiva hacia su formación académica. (p. 2).

Asimismo, Mora (2007, p. 2) afirma que es necesario realizar una reforma educativa donde el aprendizaje esté centrado en el alumno y éste aprenda a aprender. Esta reforma tiene un carácter de urgencia, especialmente en algunas materias que se han caracterizado por resistirse al cambio en cuanto a metodologías y estrategias de enseñanza.

Ante esta problemática, el mismo autor expone sobre el contexto latinoamericano: 
En las instituciones de educación superior latinoamericanas, la Enseñanza de la Física ha tenido un escaso y lento desarro1lo. [...] Por el contrario, en Estados Unidos de Norteamérica se cuenta con diversos programas de posgrado en Enseñanza de la Física, además de tener una sólida y consolidada sociedad de profesores de Física que impulsan nuevos métodos modernos de enseñanza, tales como los Workshop Physics, The Physics Education Groups, Physics by Inquiry, Tutorials in Physics, Peer Instruction, etc. (Mora, 2007, p. 4).

Además, Picquart (2007) apunta que

(...) las graves deficiencias de la educación secundaria y preparatoria en México con una enseñanza demasiada enfocada hacia la memorización en detrimento del razonamiento, cuestionan nuestro propio desempeño, y el modelo de enseñanza centrada en la clase magistral del profesor, y más aún la actitud de quien se opone al cambio. (p.30).

A raíz de esto, docentes universitarios externan su preocupación debido a la falta de conocimientos básicos en Física con que los estudiantes inician sus estudios universitarios. Picquart (2007) discutiendo sobre la situación de la enseñanza de Física en México, comenta sobre los profesores universitarios en Física: "Es muy frecuente oír a nuestros colegas quejarse de la falta de madurez, falta de métodos de estudio, de la pobrísima preparación anterior de los alumnos, que no tienen el nivel, etc.” (p. 30).

Lo anterior implica que las secuelas de las deficiencias formativas en esta ciencia y su impacto negativo en la calidad del aprendizaje se hacen evidentes cuando los estudiantes de secundaria ingresan a la universidad. Por lo tanto, esta situación puede acarrear diversos problemas a los estudiantes durante el avance en los estudios superiores.

\section{Marco teórico: Contexto de la enseñanza de la Física en Costa Rica}

La Física es una ciencia significativa e influyente que nace de la observación de la naturaleza; sin embargo, el panorama de su enseñanza indica que es necesaria la aplicación de estrategias didácticas que faciliten en los y las aprendientes la construcción de relaciones entre lo que se les enseña y los fenómenos naturales y observables cotidianamente. 
En Costa Rica, la problemática en los procesos de enseñanza y aprendizaje de esta ciencia existe de forma muy similar a lo expuesto. Las consecuencias de ese estancamiento en los procesos educativos en la materia se palpan desde la educación secundaria hasta las aulas universitarias.

A pesar de la importancia de la Física y su relación estrecha con el mundo circundante, a nivel de secundaria, la perspectiva general indica que son muchos los estudiantes que presentan dificultades con la materia. Les resulta difícil encontrar utilidad de los contenidos estudiados en las clases de Física y no demuestran interés por la materia.

Según los resultados de las pruebas de bachillerato de Ciencias del 2000 al 2007, (datos proporcionados por el Ministerio de Educación Pública), un porcentaje menor al $12 \%$ del total de estudiantes que realizaron las pruebas, eligió la materia de Física. Entre las tres ciencias (Física, Química y Biología), la Física es la que menos estudiantes eligieron dentro de este periodo.

Aún más importante es destacar la enorme diferencia en la cantidad de estudiantes que escogieron Biología en estos años, porcentaje que supera el $74 \%$ en este periodo. En general, tanto la población estudiantil como los docentes en este nivel, conocen que los temas por abarcar para prepararse para la prueba de bachillerato en Biología en su mayoría son de naturaleza teórica y más extensos, en contraste con los que se abarcan para la prueba de Física, en la que los estudiantes deben aplicar destrezas teórico-prácticas para resolver los problemas que se presentan. A pesar de esto, Física sigue siendo la ciencia que los estudiantes menos eligen para realizar en las pruebas de bachillerato.

Por otro lado, según datos publicados en La Nación (Villegas, 2008, p. 5), el porcentaje de aprobación de Física en bachillerato para el 2008 , fue del 76,7\%, siendo la materia con menor porcentaje de aprobación. Ante esta noticia, el Ministro de Educación, Leonardo Garnier (Villegas, 2008, p. 5), responde a una pequeña entrevista que se publicó y que se transcribe a continuación:

¿Cómo califica la promoción de bachillerato de este año?

Es normal, muy similar a la de años anteriores. La diferencia de este año con el anterior no es estrictamente significativa. 
¿Por qué Matemática y Física siguen siendo las materias con menor aprobación?

Primero hay un tema de cultura, desde que el alumno llega a la clase y el profesor dice que la mayoría no saldrá bien. También lo hacen los padres de familia y los mismos estudiantes piensan que en esa materia se quedarán. El temor y la angustia causan fracaso. ¿Habrá medidas para verificar que los profesores que imparten estas materias están capacitados para ello?

Aplicaremos pruebas de diagnóstico, como en Inglés. Por mucho tiempo se ha creído que si a alguien se le da Matemática y luego Pedagogía tenemos un profesor, pero no es así. Tiene que saber enseñar Matemática y requerimos el esfuerzo de las universidades para ello.

Asimismo, Andrey (2009) en un artículo publicado en el periódico La Nación, expone que los estudiantes que ingresan a las instituciones de enseñanza superior lo hacen con fuertes deficiencias en el área de Física y destaca que tienen miedo de aprender: "¿Por qué tienen miedo de la materia que explica el comportamiento de la naturaleza? ¿Será quizás porque desde su primer contacto con la Física el profesor no les inculca pasión y deseo de conocer el comportamiento de nuestro entorno?". (p. 28A).

Además, se evidencia en ambientes de formación de docentes en el área de la Física que los estudiantes, futuros docentes, aún reciben clases basadas en las metodologías tradicionalistas y por ello, es usual que se repitan estos patrones al momento de laborar como docentes de secundaria.

Esta situación ha resultado en una especie de ciclo negativo que afecta la educación de los estudiantes en el campo de la Física. Esta aseveración se explica en el hecho de que muchos de los estudiantes de secundaria no logran un aprendizaje de calidad o no se promueve la construcción de aprendizajes significativos en la materia; en ocasiones porque el docente no ha recibido una adecuada formación en didáctica, estrategias para el aprendizaje y uso de tecnologías para apoyar los aprendizajes específicamente en esta ciencia. Por lo tanto, no ha desarrollado las habilidades necesarias para desempeñarse como un buen facilitador en la materia.

Ante esta situación, se hace evidente el hecho de que la educación secundaria en el área de la Física se ve afectada, entre otros factores, 
cuando la formación universitaria de los docentes en Ciencias Naturales no está actualizada; cuando la formación de los futuros docentes no responde a las necesidades de la sociedad y de los estudiantes en particular.

Asimismo, la calidad de la educación en esta área sufre consecuencias negativas cuando existen carencias o falta de opciones en procesos de actualización docente y formación permanente, específicamente para esta área.

Por todo esto, se hace urgente encontrar soluciones para cambiar el tradicionalismo en los procesos de enseñanza-aprendizaje de la Física, y para mantener actualizados a los docentes sobre aspectos de didáctica en esta materia.

Partiendo de esta situación, para el proyecto del cual se deriva este artículo fue necesario realizar un pequeño sondeo que permitiera caracterizar la problemática en el contexto costarricense. Es importante mencionar que dicha investigación no tiene carácter exhaustivo en la definición de la problemática, debido a lo limitado de la muestra y la diversidad de factores que pueden influir en los procesos formativos en la materia. Su intención fue recopilar información relevante para el proyecto, con el objetivo de elaborar una propuesta viable que contribuya con atención al problema descrito.

\section{Metodología}

\section{Sondeo sobre los procesos de enseñanza-aprendizaje de la Física en Costa Rica}

Debido a la poca información documentada en Costa Rica sobre el problema planteado, fue necesario realizar una pequeña investigación tipo sondeo, con el objetivo determinar algunas de las causas que fundamentan, en el país, las dificultades de los estudiantes de secundaria para aprender Física y que están relacionadas con la práctica docente que se realice.

El sondeo o encuesta de opinión "es un método sistemático por medio del que se recolecta información mediante preguntas de un grupo seleccionado de personas" (Hernández, Fernández y Baptista, 2006, p. 2). Las preguntas generalmente se organizan en un cuestionario, por medio del cual se recopila la información. (Hernández et al., 2006a, p. 2).

En este caso, la encuesta tiene finalidad descriptiva y correlacional-causal; descriptiva porque pretende caracterizar a la población 
meta, proveer un perfil del fenómeno y documentar información que confirme los datos previos del planteamiento (Hernández et al., 2006 p. 3). Además se considera correlacional-causal porque busca caracterizar relaciones entre variables y vincular diferentes cuestiones dentro de la situación planteada (Hernández et al., 2006 a p. 3).

Por el gran número de individuos que componen el universo, la muestra que se utilizó no es representativa. Sin embargo, los datos recopilados fueron fundamentales para la justificación y fundamentación del proyecto. Asimismo, la comisión evaluadora del proyecto emitió la recomendación de realizar publicaciones derivadas del mismo, con el objetivo de que la información estuviera a disposición pública y pudiera ser utilizada como referencia de futuros proyectos sobre didáctica de la Física.

Se realizaron varias encuestas a distintos grupos de personas involucradas en la problemática analizada: 1) Profesionales que por su experiencia y formación se consideran especialistas en el campo de la enseñanza de las Ciencias Naturales o la Física. 2) Docentes graduados en Enseñanza de las Ciencias Naturales que, en el momento de responder la encuesta sean profesores de Física activos. 3) Estudiantes universitarios que se encuentren estudiando Enseñanza de las Ciencias Naturales. 4) Estudiantes de secundaria de cuarto y quinto año que, en el momento de responder la encuesta, estén llevando lecciones de Física en instituciones educativas de educación formal.

Las encuestas se aplicaron de forma escrita entre octubre del 2008 y agosto del 2009. Los instrumentos aplicados se pueden consultar en la sección de anexos dentro del texto completo del proyecto de graduación mencionado en la introducción, el cual se encuentra en la Biblioteca Central de la UNED.

A continuación se exponen los principales resultados de las encuestas, organizados por grupos encuestados.

\section{Resultados}

\section{De acuerdo con los especialistas}

El grupo está conformado por una muestra de cinco profesionales en el área de la enseñanza de las Ciencias, quienes se consideran especialistas en la enseñanza de la Física por su experiencia como docentes de la materia o formadores de futuros profesionales en el área. 
Según los resultados, los encuestados afirman que los estudiantes de secundaria en Costa Rica consideran la Física como una materia difícil. Se menciona dentro de su explicación que la dificultad con la que los estudiantes puedan calificar la Física depende en gran parte del profesor, es decir, de la forma como imparten las lecciones y de su preparación en la materia.

Además, mencionan dentro de los factores que contribuyen al fracaso escolar en la materia: la falta de motivación, la disociación de lo que se aprende con la vida cotidiana, falta de una adecuada didáctica en la materia, carencia de conocimiento de metodologías apropiadas para la enseñanza de la Física, deficiencias en la preparación del docente, falta de acciones correctivas ante la situación y predisposición negativa por parte de los estudiantes.

Como se observa, todos los especialistas mencionan causas que dependen directa o indirectamente del docente y algunas otras, donde éste podría ayudar a mejorar a partir de sus intervenciones; por ejemplo la motivación y disposición que los estudiantes tengan hacia el aprendizaje en la materia, condición fundamental para lograr un aprendizaje significativo.

Uno de los puntos que se destaca dentro de las respuestas, es que los docentes no están siendo formados satisfactoriamente en aspectos didácticos propios de la enseñanza de la Física. De lo anterior resulta que, en general, los conceptos teóricos de los temas que se desarrollan en secundaria son sintetizados y expresados de forma textual; tradicionalmente se enseña para que los estudiantes memoricen cada concepto. Por ello, el estudiante no logra relacionar conceptos que se le están presentando con su aplicación en el mundo y las situaciones cotidianas que se observan en la naturaleza.

Dentro de las principales carencias que han detectado en la formación de docentes de Ciencias en las instituciones de educación superior, se encuentra la falta de preparación adecuada sobre metodologías para enseñar o la poca creatividad en ellas. Al respecto, los encuestados mencionan que al futuro docente no se le prepara para la realidad por enfrentar, pues la formación en aspectos de docencia y pedagogía es insuficiente.

Ante esta situación, todos los especialistas encuestados coinciden en que la metodología predominante en las clases de Física es de tipo conductista, magistral, que utiliza la resolución de problemas en la pizarra. Sin embargo, cabe mencionar que uno de los encuestados destaca que él tiene conocimiento de educadores que utilizan una metodología 
contextualizada a sus centros, haciendo uso de los recursos tecnológicos con que cuentan e implantando giras educativas.

Por otra parte, según la opinión de los encuestados, si se innovara en la enseñanza de la Física se obtendrían resultados muy positivos en los procesos de aprendizaje de personas. Por ejemplo, se podría lograr que el estudiante descubra la relación entre la Física y lo cotidiano; que vea la Física como algo presente en todo lo circundante.

De esta forma, según indican los encuestados, las clases serían más atractivas y significativas, se lograría así un clima favorable para el aprendizaje. Asimismo, permitiría que los estudiantes se familiaricen con diversas herramientas tecnológicas y aprendan con ayuda de éstas.

Según uno de los especialistas, dentro de los principales problemas que enfrentan actualmente los docentes de Física es que su formación profesional se enfoca a la docencia en el área de Ciencias en general y no se les brinda formación especializada en la enseñanza de la Física. Según el entrevistado, es necesario formar docentes en cada uno de los campos de manera diferente.

Apunta además, que debería haber una relación más estrecha entre lo que se enseña en los colegios y lo que el docente aprende en su formación universitaria. Afirma que es preferible que el docente aprenda bien lo que necesita enseñar y no se trate de profundizar en muchos más contenidos que no va a desarrollar en las lecciones.

Asimismo, cuatro de los cinco especialistas concuerdan en que a los estudiantes de Enseñanza de las Ciencias no se les prepara adecuadamente en cuanto a estrategias didácticas. Además, algunos agregan que las estrategias que se les enseña son muy pocas o muy antiguas y, generalmente, éstas no responden al constructivismo.

Otros problemas relacionados con la formación docente en Física que fueron mencionados son: la carencia de metodologías apropiadas, desconocimiento en el campo de la investigación, falta de conocimiento de la materia y problemas de comunicación entre el docente y los estudiantes.

Según los encuestados, por medio de la diversidad de estrategias didácticas en las clases y cursos de Física, se puede mejorar la calidad de la educación en la materia y facilitar un aprendizaje significativo por parte de los estudiantes, por lo que resulta necesario capacitar y mantener actualizado a los docentes en estos aspectos.

De acuerdo con los encuestados, la diversidad de estrategias permitiría que el aprendizaje se dé en un ambiente dinámico, en el cual 
éstas serían promotoras de interacción, creatividad, trabajo de equipo y autonomía para proponer, sugerir y construir.

Además, advierten que, al diversificar las estrategias utilizadas dentro de las clases y cursos de Física, los estudiantes pueden interesarse más en la materia, comprender y asimilar mejor los nuevos conceptos, y por lo tanto, asociarlos con la vida cotidiana. También afirman que permitirían hacer sentir al estudiante más seguro con sus conocimientos en la materia.

Finalmente, los resultados concuerdan en que la tecnología puede utilizarse como un importante aliado en la actualización y formación permanente de los docentes. Según la especialista Marjorie Valverde Rojas "la tecnología hoy en día es muy importante para diversificar la práctica docente y permite además insertar a los estudiantes al ámbito actual." Además, los expertos mencionan que la tecnología puede contribuir en la actualización de los docentes de diversas formas: manteniéndolos informados sobre las diferentes temáticas que desarrolla en clase, sobre aspectos didácticos y metodológicos, teniendo acceso a diverso material para preparar sus clases, para conocer nuevas formas de presentar el conocimiento a los estudiantes. Sin embargo, el encuestado Gustavo De Lemos Morales menciona que es importante considerar la falta de acceso a las TIC que tienen muchos de los docentes del país.

\section{De acuerdo con los docentes de Física}

El segundo grupo que se encuestó está conformado por cinco docentes de secundaria que en el momento de responder la encuesta fueran docentes de Física activos.

De las respuestas obtenidas sobre las estrategias que los encuestados utilizan en las lecciones, se puede inferir que solo algunos docentes se interesan en asuntos como la planificación de las lecciones, la utilización de diversas estrategias para diferentes estilos de aprendizaje, la propuesta de clases motivadoras, entretenidas y significativas, entre otras.

Según los resultados, tres de los docentes aseguran no haber recibido formación sobre didáctica de la Física y estrategias para enseñar esta materia durante su carrera como estudiantes de la Enseñanza de las Ciencias. Asimismo, dos de los encuestados aseguran que tampoco recibieron formación sobre el uso de tecnologías aplicadas a los procesos de aprendizaje. 
Por otra parte, los resultados indican que no en todas las instituciones educativas hay atención a la capacitación y la actualización de los docentes. Esto evidencia la necesidad de que los docentes reciban actualización en aspectos de didáctica de la materia y nuevos recursos para utilizar en el aprendizaje de ésta.

Todos los encuestados concuerdan en que si tuvieran más conocimientos sobre estrategias innovadoras en la didáctica de la Física, los estudiantes podrían mejorar sus aprendizajes. Así, afirman que les gustaría ampliar sus conocimientos acerca de estrategias didácticas para desarrollar en sus clases.

\section{De acuerdo con los estudiantes de Enseñanza de las Ciencias Natu- rales (futuros docentes)}

El tercer grupo de encuestados está conformado por 22 estudiantes universitarios de Enseñanza de las Ciencias Naturales, de nivel de bachillerato de las siguientes universidades: Universidad Estatal a Distancia (UNED), Universidad de Costa Rica (UCR) y Universidad Americana (UAM).

Resulta importante mencionar que de esta muestra, el 91\% se encontraba trabajando como docentes en el área de las Ciencias Naturales en el momento de responder la encuesta. De esta población que trabaja solamente un $15 \%$ imparte clases de Física.

Dentro de los resultados obtenidos un 59\% de los encuestados afirma que la metodología de los cursos de Física que ha recibido en su formación universitaria es de tipo magistral, con estrategias de trabajo individual en su mayoría. En general, las actividades que se proponen se limitan a la resolución de ejercicios que requieren del manejo y aplicación de datos y fórmulas. No se propician las actividades de análisis y la reflexión que faciliten la apropiación de los conceptos, más allá de la aplicación de fórmulas para la resolución de problemas. Otro $23 \%$ contestó que la metodología es magistral, pero acompañada de laboratorios participativos. Sin embargo, se ha constatado que no todas las universidades cuentan con laboratorios de Física.

Sobre este aspecto, resulta importante discutir que el equipo que se utiliza en la mayoría de las prácticas de laboratorio en los cursos de Física nivel universitario resultan costosos; por la misma razón, son escasos los colegios que cuentan con laboratorios de este tipo. Así, las 
prácticas y ejercicios que los estudiantes universitarios de Enseñanza de las Ciencias Naturales desarrollan en los laboratorios de los cursos de su carrera, no siempre pueden ser aplicados en su labor como docentes de secundaria. Por lo tanto, se hace necesario incluir dentro de los contenidos de los cursos prácticos de la carrera, estrategias para la realización de demostraciones y experimentos mediante objetos de fácil acceso, sin necesidad hacer uso de instrumentos y equipo especializado.

Por otra parte, los resultados muestran que un $77 \%$ de los encuestados considera que no se les está formando adecuadamente en el área de didáctica de las Ciencias. Además, igual porcentaje afirma no estar satisfecho con la formación en estrategias y recursos didácticos que ha llevado en los cursos de didáctica de las Ciencias.

A lo anterior se une, según la opinión del $64 \%$ de los encuestados, que es en Biología en la que se enfatizan más los aspectos didácticos, lo que parece evidenciar que la didáctica específica de la Física recibe una menor atención. En una perspectiva general, esta situación podría estar contribuyendo a la problemática descrita en las primeras secciones de este artículo.

Por otra parte, se observa que un $86 \%$ de los encuestados no se encuentran satisfechos con la formación que han recibido hasta ese momento, en cuanto al uso de tecnologías como recursos didácticos o educativos. Finalmente, el 95\% responde que sí le gustaría recibir mayor formación sobre estrategias didácticas aplicadas a la enseñanza de la Física.

\section{De acuerdo con los estudiantes de secundaria}

El cuarto grupo de encuestados está conformado por 32 estudiantes de secundaria de cuarto y quinto año, de colegios públicos y privados ubicados en Coronado, Palmares y Guápiles.

La primera parte de la encuesta se elaboró con preguntas cerradas, con el propósito de recopilar información sobre la apreciación que tienen los estudiantes sobre sus procesos de aprendizaje en Física y su actitud hacia la materia.

En esta sección, un 34\% de los estudiantes respondió que solo en algunas ocasiones se siente motivado para aprender durante las lecciones, a los que sigue un $28 \%$ de los estudiantes que afirma casi nunca se siente motivado. Por lo tanto, más de un $60 \%$ de las respuestas 
evidencian que es necesario mejorar los niveles de motivación de los estudiantes por aprender Física.

Por otra parte, los resultados revelan que en su mayoría, los estudiantes solo ocasionalmente recuerdan lo estudiado y los conocimientos aplicados para una prueba, después de realizar la misma. Además, la mayoría de los estudiantes admite que nunca o casi nunca siente deseo de estudiar o investigar fuera de clases sobre la materia. Asimismo, un $44 \%$ de los encuestados afirma que sólo en ocasiones encuentran agradables las clases de Física. Estos tres aspectos trazan focos de análisis acerca de si realmente se está logrando un aprendizaje significativo en la materia, además del hecho que no se evidencia un interés por explorar o investigar sobre esta ciencia por parte de los aprendientes.

Finalmente, en una escala de 1 a 10 siendo 1 el puntaje que indica la menor dificultad y 10 el puntaje que indica la mayor dificultad, un $25 \%$ de los encuestados califica la materia con un 5, mientras que un $31 \%$ la valora con un puntaje de 8 a 10. Esto manifiesta que son más los estudiantes que sienten que la materia es difícil, que quienes la califican como una materia fácil.

La segunda parte de la encuesta pregunta sobre las estrategias y la forma como él o la docente imparte las clases. En esta sección, los resultados indican que la mayoría de encuestados opinan que sólo ocasionalmente el docente contribuye con su proceso de aprendizaje mediante la forma en que imparte las lecciones. Asimismo, se observa que la gran mayoría de los encuestados opina que el profesor casi nunca o nunca utiliza diversidad en las estrategias o actividades desarrolladas en las clases.

Un alto porcentaje de estudiantes afirma que el educador casi nunca o nunca propone clases que hagan que el desarrollo de los temas sea entretenido. Además, el $44 \%$ de los encuestados opina que el docente casi nunca o nunca contribuye a que se sientan motivados.

Finalmente, en esta parte de la encuesta se hace evidente que los estudiantes califican de diversas maneras la labor del docente en relación con la metodología y estrategias que utiliza.

Los resultados de la tercera parte de la encuesta, permiten comprobar la preferencia de los estudiantes por la Biología, como la ciencia en la cual tienen planificado o ya han seleccionado presentar la prueba de bachillerato. 
En la cuarta parte, los resultados permiten corroborar que los aprendientes consideran que la manera de impartir lecciones del docente (forma de comunicarse, de explicar y de planificar las clases) resultan elementos claves que influyen en el logro o no de su aprendizaje.

Un $97 \%$ de encuestados afirma que la utilización de más estrategias y actividades diversas, mejorarían el aprendizaje y motivación por la Física. Además, mencionan que propuestas más dinámicas mejorarían las clases en esta ciencia.

\section{Análisis y recomendaciones}

A partir de la investigación bibliográfica desarrollada y el sondeo realizado, se evidencia que la problemática del aprendizaje de la Física en el nivel de secundaria en Costa Rica existe y que tiene características similares al panorama global que plantean autores reconocidos en el tema.

Se concluye que las necesidades de los educandos en el área de la Física deben ser atendidas mediante un cambio en la planificación y ejecución de las estrategias utilizadas en las lecciones.

Resulta necesario acercar a los aprendientes a los contenidos de una forma cotidiana; desde el mundo que ya conoce, mediante interrogantes y situaciones que despierten la curiosidad y su necesidad por la exploración. De esta forma, se podrá partir de sus conocimientos previos hacia lo nuevo. Además, los y las aprendientes requieren apropiarse de la importancia y la utilidad de las temáticas que se desarrollan en clase.

Por ser una ciencia exacta en donde se mezcla lo teórico y lo experimental, para aprender Física es primordial se abran espacios en los que la comunidad estudiantil pueda reflexionar, analizar y discutir sobre las diversas temáticas desarrolladas en las lecciones. El dinamismo cobra especial relevancia cuando facilita que el aprendiente rompa con la rutina de las clases tradicionales, despertando su interés y aumentando su motivación por aprender de esta ciencia.

Por consiguiente, el educador en Física necesita planificar cada lección como una vivencia nueva, que rete al estudiante a crear nuevas relaciones entre conceptos, a reestructurar lo que ya conoce y ampliar sus estructuras mentales. Todo esto, permitirá que los conocimientos se conviertan en nuevas herramientas para quien aprende, útiles en su vida cotidiana y en su futuro profesional. 
Según McDermott y Shaffer (s.f.), el proceso de aprendizaje en Ciencias para jóvenes se hace más efectivo cuando las bases para la construcción de los conceptos científicos se establecen mediante experiencias concretas.

Ante esto, las tecnologías de la información y la comunicación son un medio que facilita el logro de este propósito, así como que el proceso educativo pueda ser disfrutado por quienes participan en él. Las múltiples aplicaciones informáticas y web desarrolladas para el aprendizaje de la Física deberían ser aprovechas para la construcción de aprendizajes significativos, para el desarrollo de experimentos virtuales y la exploración de entornos a los que de una manera real, sería muy difícil acceder.

Además, la diversidad de estrategias didácticas, así como la variedad en las actividades de mediación que se propongan dentro de los salones de clase, permitiría, indudablemente, mejorar la disposición de los estudiantes para aprender, por lo que el proceso de enseñanza-aprendizaje sería más significativo, así como entretenido y agradable, tanto para los estudiantes como para el docente mismo.

Por ejemplo, existen instituciones que están implantando algunas estrategias para solventar dichas necesidades. Por ejemplo, en México Picquart (2007) propone realizar un cambio en los cursos de Física que imparte dicha institución. Ante esto, afirma que

este cambio incorpora la detección de las ideas previas de los alumnos y la puesta en marcha de estrategias didácticas específicas para lograr el cambio conceptual adecuado. [...] Se enfatiza también sobre la necesidad de una formación pedagógica del profesorado. (p. 29).

Otro ejemplo de este esfuerzo lo está haciendo actualmente la Universidad de Columbia Británica en Canadá, en donde se contrató a Carl Wiemann para desarrollar un proyecto destinado a "reemplazar el curso tradicional en el cual los estudiantes son llenados de información frecuentemente sin entenderla y sustituirlo por una forma más interactiva de enseñanza que permita una construcción sólida de los conceptos" (Picquart, 2007, p. 30).

Por lo tanto, es necesario facilitarle al docente las herramientas necesarias para que descubra la importancia de mantenerse actualizado 
y de aplicar estrategias diferentes e innovadoras en sus clases. Todo esto buscando que el aprendizaje se convierta en nuevas herramientas para el aprendiente, útiles en su vida cotidiana y en su futuro profesional.

\section{Conclusiones}

A partir de la investigación bibliográfica desarrollada y el sondeo realizado, se ha comprobado que varias de las causas de la problemática del aprendizaje de la Física en el nivel de secundaria tienen una estrecha relación con la labor que los educadores realizan dentro de las instituciones de formación secundaria, y por lo tanto, la manera en que planifica y desarrolla las lecciones en esta materia.

En general, cuando la acción docente se basa en una metodología tradicionalista, la cual limita la participación del estudiante e invita a reproducir una serie de conceptos teóricos y mecanismos prácticos para la resolución de problemas, la calidad de los aprendizajes de los estudiantes se ve en detrimento.

Así, se ha expuesto que, en general, la metodología tradicionalista predominante en los procesos de enseñanza y aprendizaje de la Física, supone un papel pasivo del estudiante y demanda principalmente una respuesta de memorización y repetición. No promueve el análisis, la reflexión, las relaciones entre conceptos ya incorporados y los nuevos, ni la construcción del conocimiento en la ciencia.

Se hace evidente que a pesar de que la educación en Física en algunos países de América Latina ha identificado la necesidad de realizar cambios en el campo de su enseñanza y pedagogía, la aplicación real y permanente de nuevas estrategias didácticas y metodologías educativas actualizadas aun no se ha implantado en la mayoría de las prácticas educativas a nivel de secundaria.

Se demuestra además, que la problemática también se presenta en Costa Rica, por lo que resulta necesario desarrollar estrategias que aumenten la motivación y el interés de los estudiantes por esta ciencia. Se considera esencial realizar cambios en la metodología de su enseñanza que faciliten el aprendizaje significativo de ésta, por parte de los aprendientes. Para la cual es fundamental establecer un mayor dinamismo y participación de los estudiantes dentro de las lecciones de Física.

De esta forma, se comprueba la necesidad de realizar mejoras en el planteamiento y desarrollo de las clases de Física, de manera que 
el docente logre desarrollar las lecciones como espacios interesantes y dinámicos, que despierten la curiosidad de los estudiantes, siento estos los sujetos centrales del proceso, promoviendo la exploración, la experimentación y la discusión de los contenidos.

Asimismo, se pudo comprobar la necesidad por parte de los docentes y futuros docentes del área de Física, de formarse en aspectos de didáctica en la materia, en estrategias para mediación y en la utilización de los recursos tecnológicos como herramientas educativas; todo esto para facilitar el aprendizaje significativo de la materia a nivel de secundaria.

Los profesionales en educación quienes se dedican a enseñar Física, necesitan actualizarse para implantar eficazmente las metodologías educativas actuales. Además, requieren de capacitación permanente para sacar provecho de las ventajas y facilidades que ofrecen la tecnología en la construcción de aprendizajes de calidad en esta ciencia.

Se fundamenta la necesidad de realizar acciones correctivas e implantar planes que permitan preparar de una forma más adecuada a los futuros docentes de Física, a partir de la revisión y actualización de los programas de las carreras en Enseñanza de la Ciencias Naturales y los cursos respectivos. Además es importante prestar mayor atención a aspectos de didáctica y mediación pedagógica específicas para esta ciencia, así como preparar a los futuros docentes para una adecuada utilización de tecnologías para apoyar el aprendizaje de la Física. Por otra parte, se considera fundamental que se propongan cambios en la metodologías que se utilizan en los cursos que ofrecen las universidades relacionadas con la materia, de manera que el futuro docente pueda experimentar en su propio proceso formativo, los beneficios de nuevas estrategias y metodologías actuales.

Al mismo tiempo, es necesario desarrollar propuestas de actualización y capacitación a las que tengan acceso quienes ya son egresados y laboran como docentes, con el propósito de solventar las carencias en su formación y promover con una cultura de actualización constante. 


\section{Referencias bibliográficas}

Andrey, O. (2009, enero 6). Pedagogía en física. La Nación. Recuperado de http://wvw. nacion.com/ln_ee/2009/enero/06/opinion1829121.html

Hernández, R., Fernández, C. y Baptista, L. (2006a). Encuestas (Surveys). En Metodología de la investigación (Cap 6.) [CD]. D.F.: Mc-Graw-Hill e Interamericana Editores S.A.

McDermott, L. \& Shaffer, P. (s.f). Preparing teachers to teach physics and physical science by inquiry. Recuperado de http://quarknet.fnal.gov/fellows/TLDownloads/modermott_shaffer.pdf

Mora, C. (2007). Lapen y la formación de profesores de física. Recuperado de http:// www.fisica.ucr.ac.cr/varios/ponencias/lapen.pdf

Picquart, M. (2007). ¿Qué podemos hacer para lograr un aprendizaje significativo de la Física? Recuperado de http://journal.lapen.org.mx/jan08/LAJPE-120\%20 Final\%20Picquart.pdf

Villegas, J. (2008, diciembre 19). Solo 43\% de los aspirantes al bachillerato logran graduarse. La Nación, pp. 5A. 